\title{
Influence of fastening conditions on the deformation of a metal sample when it is stretched
}

\author{
Valeriy Prokopiev ${ }^{*}$, Tatiana Zhdanova, and Nerses Simonyan \\ Moscow State University of Civil Engineering, Yaroslavskoe shosse, 26, Moscow, 129337, Russia
}

\begin{abstract}
Investigates the effect of conditions for fixing a metal thin plate on its deformation under tension. At observance of GOST on fixing the edges of the plate in a tearing machine, the greatest thinning occurs in the middle along the length of the sheet.
\end{abstract}

\section{Introduction}

In the process of designing and manufacturing various metal elements of building structures, machines and mechanisms, elasticity, viscosity and plasticity are fundamental. Their characteristics are determined by testing samples for static loads, dynamic, cyclic, and pressure testing. The test results allow you to determine the optimal size in the design process of elements, which leads not only to practical but also economic benefits.

One of the most important methods for determining the properties of a metal is a tensile test to determine its strength. The sample is tested by a bursting machine. Such tests allow to determine the Young's modulus, the elastic limit of the metal.

When a metal is overstressed, the sample begins to elongate without mechanical impact with the formation of a transverse narrowing and subsequent rupture in this place. As a rule, the gap occurs in the middle of the sample along the length. In this case, the clamps of the bursting machine should be the same, the sample should be fixed without distortions and have dumb-bells on the ends.

Different metals and alloys have different mechanical and technological properties, which predetermine the quality of machine parts, as well as the workability of the metal. Therefore, the relevant samples are subjected to other tests for compression, bending, hardness, fatigue strength, etc. Test methods are governed by the relevant State Standards.

The paper investigates the influence of the conditions for fixing a metal thin sheet on its deformation. It is believed that a thin-walled plate is subject to a tensile longitudinal force uniformly distributed over the entire lower edge. Plate dimensions and test conditions must comply with the normative document [1]. In case of violation of the requirements of this regulatory document, in particular the conditions of fixing in a tensile machine, the sheet may break not in the middle.

\footnotetext{
* Corresponding author: viprokopiev@ mail.ru
} 


\section{Description of a mathematical model}

The stress state of a stretchable plate as a three-dimensional elastic body is described by the Lamé equations [2]:

$$
\mu \Delta u_{i+}(\lambda+\mu)\left(\partial e / \partial x_{i}\right)=f_{i}, \quad i=1,2,3 ;
$$

where $\lambda, \mu$ - the Lame coefficients, $\mathrm{e}-$ the volume deformation, $f_{i}$ - the components of the vector of external forces at a point, $\Delta-$ the Laplace operator:

$$
\Delta=\partial^{2} / d x^{2}+\partial^{2} / d y^{2}+\partial^{2} / d z^{2}
$$

Unknown in equations (1) are displacements of $u_{i}$ along the coordinate axes $x-u x, y-$ $u y, z-u z$.

Three variants of the conditions for fixing the sample plate in a tensile machine are considered:

1 - fixing according to GOST - the same conditions for fixing the upper and lower edges of the sample $u=v=w=0$;

2 - not fixed in accordance with GOST - on the upper edge of the sample $u=v=w=$ 0 , on the lower edge $w=0, u$ and $v$ are allowed to move;

3 - fixing not according to GOST - identical conditions for fixing the upper and lower edges of the sample $w=0, u$ and $v$ movements are allowed.

Equations (1) with such variants of constraints are Dirichlet boundary value problems, for the solution of which the finite element method is used. For calculations, the software package SCAD Office version 21.1 [3] was selected.

The geometric dimensions of the sample and the mechanical characteristics of the material are assumed to be:

- plate length $250 \mathrm{~mm}$ with cross-section dimensions of $50 \mathrm{~mm}$ along the $\mathrm{x}$ axis and 10 $\mathrm{mm}$ along the y axis;

- plate material: steel $(\mathrm{E}=2.1 \times 105 \mathrm{MPa}, v=0.3)$;

- the load is $5000 \mathrm{MPa}$ on the edges;

- computer simulation was carried out with 8-node isoparimetric finite elements (FE dimensions equal to $2.5 \times 2.5 \times 2.5 \mathrm{~mm}$ ).

\section{Comparison of the obtained results}

The following results were obtained.

Option 1 fastening edges. The greatest displacements of the grid nodes in the cross section of the plate with the formation of a "waist" are realized in the middle along the length of the plate (Figure 1). 


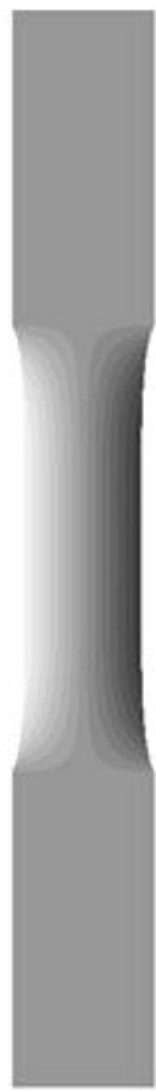

a)

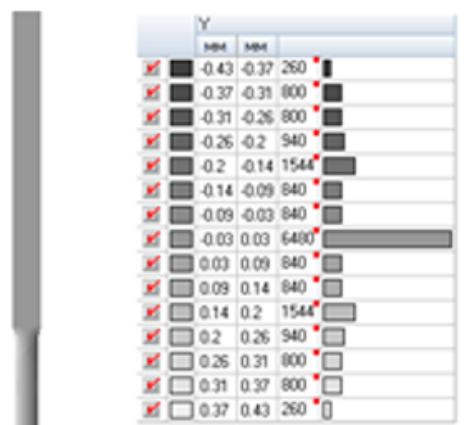

b)

Fig. 1. Horizontal movements: a) across plate width, b) on plate thickness.

Option 2 fastening edges. The reduction of the "waist" is realized from the top edge of the plate to the bottom (Figure 2). 

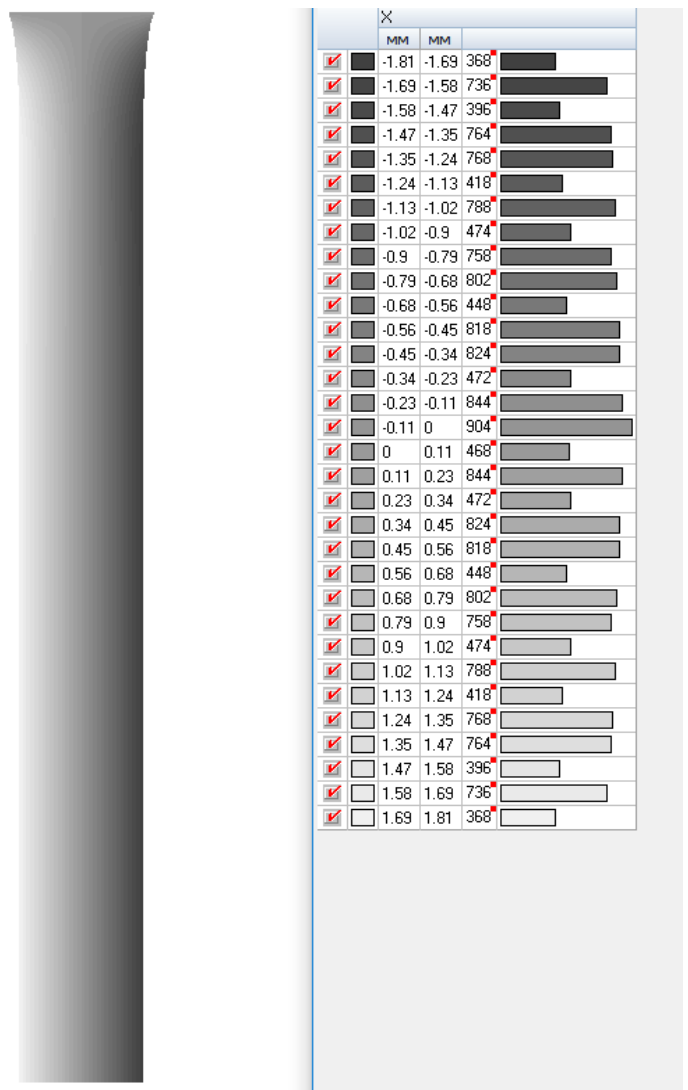

a)
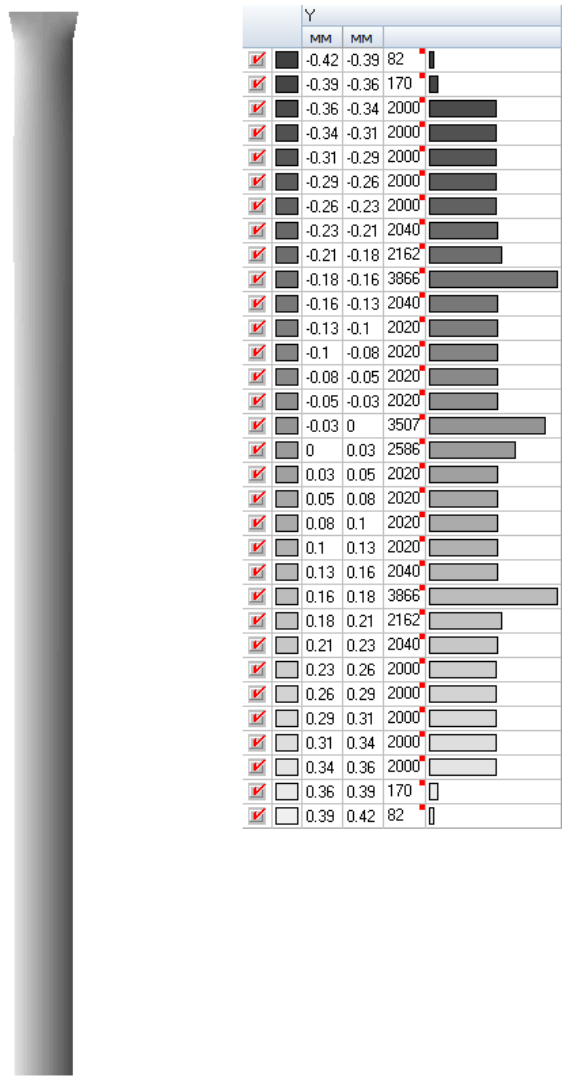

b)

Fig. 2. Horizontal movements: a) across plate width, b) on plate thickness.

Option 3 fastening edges. The "upper" edge of the plate is fixed with a "sliding seal". All sections of the plate along its entire length are deformed in exactly the same way.

\section{Conclusions}

Three options of boundary fixation were investigated. All options give results with largest deformation at the center of the plate.

First result show than GOST rules of fixation give some boundary nonphysical effect through vertical edge of plate.

Second and third fixations is recommended for computational verification of tensile test.

\section{References}

1. GOST 11701-84 Metals. Methods of tensile testing of thin sheets and strips.

2. V. N. Sidorov, V. V. Vershinin The finite element method in the calculation of structures. Theory, algorithm, examples of calculations in the software package SIMULA Abaqus. Textbook (Association of Construction Universities, Moscow, 2015) 
3. V. S. Karpilovsky, E. Z. Kriksunov, A. A. Malyarenko, A. V. Perelmuter, M. A. Perelmuter. Computing complex SCAD. (Association of Construction Universities, Moscow, 2008) 